\title{
Antidepressant Effects of a Single Dose of Ayahuasca in Patients With Recurrent Depression
}

\author{
A SPECT Study \\ Rafael Faria Sanches, PhD, *† Flávia de Lima Osório, PhD, *t Rafael G. dos Santos, PhD, * \\ Ligia R.H. Macedo, MSc, * João Paulo Maia-de-Oliveira, PhD, *ł\$ Lauro Wichert-Ana, PhD, * \\ Draulio Barros de Araujo, PhD, // Jordi Riba, PhD, †\#**t† \\ José Alexandre S. Crippa, PhD, * + and Jaime E.C. Hallak, PhD ${ }^{*}+$
}

\begin{abstract}
Ayahuasca is an Amazonian botanical hallucinogenic brew which contains dimethyltryptamine, a 5- $\mathrm{HT}_{2 \mathrm{~A}}$ receptor agonist, and harmine, a monoamine-oxidase A inhibitor. Our group recently reported that ayahuasca administration was associated with fast-acting antidepressive effects in 6 depressive patients. The objective of the present work was to assess the antidepressive potentials of ayahuasca in a bigger sample and to investigate its effects on regional cerebral blood flow. In an open-label trial conducted in an inpatient psychiatric unit, 17 patients with recurrent depression received an oral dose of ayahuasca $(2.2 \mathrm{~mL} / \mathrm{kg})$ and were evaluated with the Hamilton Rating Scale for Depression, the Montgomery-Åsberg Depression Rating Scale, the Brief Psychiatric Rating Scale, the Young Mania Rating Scale, and the Clinician Administered Dissociative States Scale during acute ayahuasca effects and 1, 7, 14, and 21 days after drug intake. Blood perfusion was assessed eight hours after drug administration by means of single photon emission tomography. Ayahuasca administration was associated with increased psychoactivity (Clinician Administered Dissociative States Scale) and significant score decreases in depression-related scales (Hamilton Rating Scale for Depression, Montgomery-Åsberg Depression Rating Scale, Brief Psychiatric Rating Scale) from 80 minutes to day 21. Increased blood perfusion in the left nucleus accumbens, right insula and left subgenual area, brain regions implicated in the regulation of mood and emotions, were observed after ayahuasca intake. Ayahuasca was well tolerated. Vomiting was the only adverse effect recorded, being reported by $47 \%$ of the volunteers. Our results suggest that ayahuasca may have fast-acting and sustained antidepressive properties. These results should be replicated in randomized, double-blind, placebo-controlled trials.
\end{abstract}

Key Words: ayahuasca, dimethyltryptamine, harmine, monoamine oxidase inhibitors

(J Clin Psychopharmacol 2016;36: 77-81)

From the*Department of Neurosciences and Behavior, Ribeirão Preto Medical School, University of São Paulo, Ribeirão Preto, Brazil; †Centre d'Investigació de Medicaments, Servei de Farmacologia Clínica, Hospital de la Santa Creu i Sant Pau, Barcelona, Spain.; \$National Institute of Science and Technology, Translational Medicine, Ribeirão Preto; §Department of Clinical Medicine, $\|$ Onofre Lopes University Hospital, $₫$ Brain Institute, Federal University of Rio Grande do Norte, Natal, Brazil; \#Human Experimental Neuropsychopharmacology, Institut de Recerca, Hospital de la Santa Creu i Sant Pau; **Departament de Farmacologia i Terapèutica, Universitat Autònoma de Barcelona; and †Centro de Investigación Biomédica en Red de Salud Mental, CIBERSAM, Barcelona, Spain

Received March 18, 2015; accepted after revision September 22, 2015.

Reprints: Rafael G. dos Santos, PhD, Departamento de Neurociências e

Ciências do Comportamento, Faculdade de Medicina de Ribeirão Preto,

Universidade de São Paulo, Hospital das Clínicas, Terceiro Andar, Av.

Bandeirantes, 3900, Ribeirão Preto, São Paulo, Brazil

(e-mail: banisteria@gmail.com).

Supplemental digital contents are available for this article. Direct URL citation appears in the printed text and is provided in the HTML and PDF versions of this article on the journal's Web site (www.psychopharmacology.com). Copyright (C) 2015 Wolters Kluwer Health, Inc. All rights reserved.

ISSN: 0271-0749

DOI: 10.1097/JCP.0000000000000436
A yahuasca is a hallucinogenic botanical brew prepared by deA coction of the bark of the vine Banisteriopsis caapi together with the leaves of the shrub Psychotria viridis. ${ }^{1}$ Banisteriopsis caapi contains $\beta$-carboline alkaloids (harmine, tetrahydroharmine, and harmaline) that act as reversible inhibitors of monoamine oxidase(MAO)-A, whereas $P$. viridis is rich in $N, N$-dimethyltryptamine (DMT), $\mathrm{n}$ hallucinogenic tryptamine that acts as a $5-\mathrm{HT}_{1 \mathrm{~A} / 2 \mathrm{~A} / 2 \mathrm{C}}$ agonist. ${ }^{1,2}$ The psychoactive effects of ayahuasca are produced by a combined action of $\beta$-carbolines and DMT: because DMT is metabolized by MAO-A in the gut and liver, peripheral MAO-A inhibition by $\beta$-carbolines allows DMT to reach systemic circulation and the brain, where it acts at frontal and paralimbic areas. ${ }^{1-3}$

Preclinical, observational, and experimental studies in healthy volunteers suggest that ayahuasca has antidepressive properties, ${ }^{4}$ and we recently reported that administration of ayahuasca to 6 patients with recurrent major depressive disorder (MDD) was associated with fast-acting and enduring antidepressive effects. ${ }^{5}$ The objective of the present work was to assess the antidepressive potentials of ayahuasca in a bigger sample and to investigate its effects on regional cerebral blood flow.

\section{MATERIALS AND METHODS}

\section{Volunteers}

Seventeen volunteers ( 14 women; mean age, 42.71 years; SD, 12.11) with recurrent MDD participated in the study. Three volunteers were experiencing a current mild depressive episode, 13 a current moderate episode, and 1 a current severe depressive episode. Participants were recruited through local advertisement and by referrals from private psychiatric clinics. Volunteers were patients that did not present a therapeutic response to their current antidepressive drug and were changing their medication. Patients were invited to participate in an experimental study investigating the antidepressive properties of ayahuasca, which is relatively well known in Brazil because of syncretic religions, such as the Santo Daime and the União do Vegetal, which consume the brew ritually. ${ }^{6}$ No payment was offered for study participation, because in Brazil this practice is not allowed. Volunteers passed a 2-week washout period before study participation, and none of them had ever used illicit drugs or ayahuasca. Participants showed no current clinical pathology or pregnancy, as assessed by medical interview, physical examination, and laboratory tests. A diagnosis of bipolar or psychotic disorder, presence of active psychotic symptoms, and a previous history of mania or hypomania induced by antidepressant or substance use were considered exclusion criteria.

Volunteers received detailed information regarding the effects of ayahuasca based on the scientific literature and on psychiatric criteria for hallucinogen-induced disorders (Diagnostic and statistical manual of mental disorders, 5th ed. American Psychiatric Association, Washington, DC. 2013). All patients gave their 
written informed consent to participate. The study was conducted in accordance with the Declarations of Helsinki and Tokyo concerning experimentation on humans and approved by the local Ethics Committee (process HC-RP 2484/2008).

\section{Drug}

The ayahuasca sample, obtained from the Brazilian religious organization Santo Daime, was the same used in our previous report and contained $0.8 \mathrm{mg} / \mathrm{mL}$ DMT, $0.21 \mathrm{mg} / \mathrm{mL}$ harmine, and no harmaline as measured by GC/MS (tetrahydroharmine was not analyzed due to a lack of analytical requirements). ${ }^{5}$ Each patient drank a single dose of 120 to $200 \mathrm{~mL}$ of ayahuasca $(2.2 \mathrm{ml} / \mathrm{kg})$, which is a dose commonly in the Santo Daime and União do Vegetal. ${ }^{6}$ Thus, the range of alkaloid content in individual doses was 96 to $160 \mathrm{mg}$ DMT and 25 to $42 \mathrm{mg}$ harmine.

\section{Medical Interview and Laboratory Tests}

A general clinical examination; a laboratory work-up consisting of a complete blood cell count; evaluation of blood glucose levels; plasma sodium, potassium, urea, creatinine, bilirubin, liver enzymes, and urinary $\beta$-HCG (in women of childbearing age); an electrocardiogram; and a clinical interview to confirm the diagnosis of MDD (Structured Clinical Interview for DSM-IV-SCID-IV) ${ }^{7}$ were performed.

\section{Assessment of Ayahuasca Effects on Psychiatric Symptoms}

Depressive symptoms were assessed with the Hamilton Depression Rating Scale (HAM-D) and the Montgomery-Åsberg Depression Rating Scale (MADRS) ${ }^{8}$ maniac symptoms with the Young Mania Rating Scale (YMRS), ${ }^{9}$ general psychiatric symptoms with the Brief Psychiatric Rating Scale (BPRS), ${ }^{10}$ and dissociative symptoms with the Clinician Administered Dissociative States Scale (CADSS) ${ }^{11}$ (see Supplementary Text, Supplemental Digital Content 1, which shows detailed information on each scale, http://links.lww.com/JCP/A344). Scales were completed by a trained psychiatrist 10 minutes before ayahuasca administration (baseline) and 40, 80, 140, and 180 minutes after drug intake. The HAM-D, MADRS, and BPRS scales were also completed 1 (D1), 7 (D7), 14 (D14), and 21 (D21) days after ayahuasca administration.

\section{Assessment of Ayahuasca Effects on Regional Cerebral Blood Flow}

The effects of ayahuasca on regional cerebral blood flow were assessed by single photon emission tomography (SPECT) imaging performed before drug intake and eight hours after treatment. This time point was chosen to assess if any observed changes in blood perfusion were still present after 4 to 6 hours, when the main active effects of ayahuasca had subsided ${ }^{2,3}$ (see Supplementary Text, Supplemental Digital Content 2, which describes the SPECT imaging methodology, http://links.lww.com/JCP/A345).

\section{Tolerability Measures}

Adverse effects were recorded by spontaneous verbal reports, and blood pressure and heart rate were measured 10 minutes before ayahuasca administration and 40,80,140, and 180 minutes after drug intake.

\section{Experimental Procedure}

Volunteers were admitted to an inpatient psychiatric unit for two weeks prior to ayahuasca administration in an open-label trial.
During this time volunteers did not take any psychiatric medication or recreational drugs. Except for the initial information on the effects of ayahuasca, there was no formal preparation sessions prior to drug administration. Since our intention was to investigate the pharmacological effects of ayahuasca, non-drug factors that are commonly present in ritualized and religious contexts, such as singing or listening to music, ${ }^{6}$ were excluded. Ayahuasca was administered in a quiet dimly lit room, where volunteers remained seated in a comfortable reclining chair. The session was performed individually and lasted $4 \mathrm{~h}$, and no psychological intervention was included after drug effects had subsided. Patients remained under observation for $24 \mathrm{~h}$ after drug intake, and if no complications were noticed they were discharged.

\section{Statistical Analysis}

After verification of data distribution, descriptive statistics and repeated-measures analysis of variance (ANOVA) were used for statistical analysis. When a significant effect was observed, pairwise comparisons were performed by means of Student $t$ tests. Significance was set at $P<0.05$.

\section{RESULTS}

\section{Ayahuasca Effects on Psychiatric Symptoms}

Average baseline score in the HAM-D scale was 19.24 (SD = 5.52 ), which is considered a moderate level of depression, and 25.6 points $(\mathrm{SD}=7.6)$ in the MADRS scale (see Supplementary Table, Supplemental Digital Content 3, which shows clinical and demographic characteristics of patients, http://links.lww.com/JCP/A346). Administration of ayahuasca was associated with significant HAM-D and MADRS score decreases from 80 to 180 minutes $(P<0.01)$ and from D1 to D21 $(P=0.000)$ (Fig. 1). At D21, average score in the HAM-D scale was $7.56(\mathrm{SD}=4.7)$, which is considered a mild level of depression.

Significant score increases in the CADSS scale were observed from 40 to 80 minutes $(P<0.01)$ after ayahuasca intake, while no significant changes in YMRS scores were observed (Fig. 1) (see Supplementary Figure, Supplemental Digital Content 4, which shows score changes in the HAM-D, MADRS, YMRS, and CADSS scales for each patient individually, http://links.lww.com/JCP/A347)

Ayahuasca administration was also associated with significant score decreases in the Anxious-Depression (from 40 to 180 minutes, $P<0.01$; and from D1 to D21, $P=0.000)$, Thinking Disorder (180 minutes, $P<0.05$; and at D1, D14, and D21,

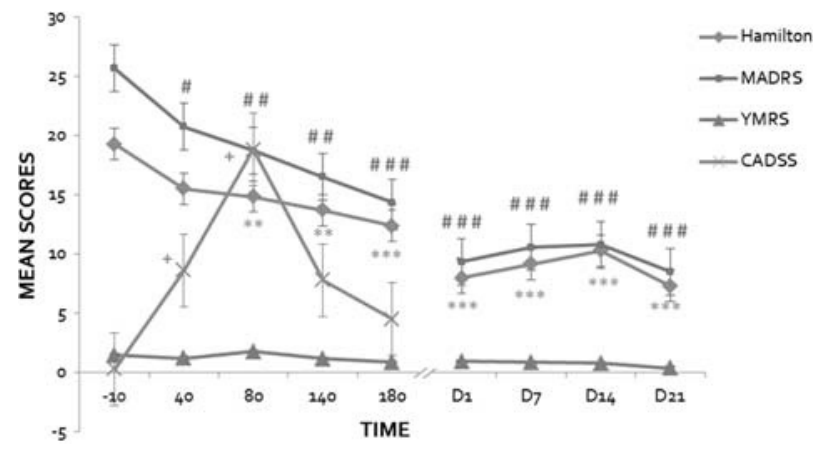

FIGURE 1. Temporal distribution of scores (mean; SD) on the HAM-D, MADRS, YMRS and CADSS scales $(n=17)$. HAM-D: ${ }^{* *} P<0.01,{ }^{* * *} P<0.001$; MADRS: $\# P<0.05, \# \# P<0.01$, $\# \# \# P<0.001$; CADSS: $+P<0.01$. Error bars denote 1 SEM. 


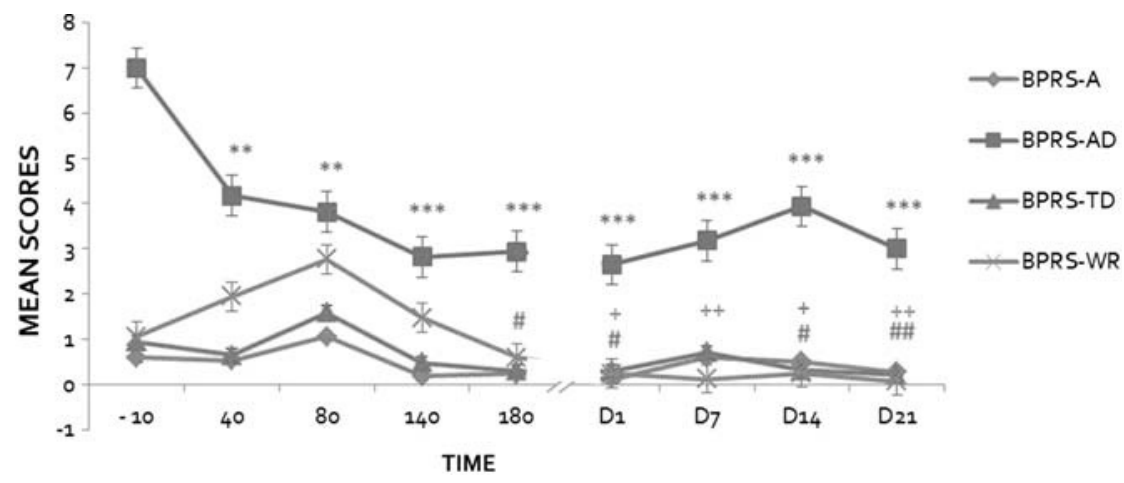

FIGURE 2. Temporal distribution of scores (mean; SD) of the BPRS subscales (A, activation; $A D$, anxious-depression; TD, thinking disorder; WR, withdrawal-retardation $(\mathrm{n}=17)$. AD: ${ }^{*} P<0.01,{ }^{*} * P<0.001$; TD: $\# P<0.05, \# \# P<0.01$; WR: $+P<0.05 ;++P<0.01$. Error bars denote 1 SEM.

$P<0.05$ ), and Withdrawal-Retardation (from D1 to D21, $P<0.05)$ subscales of the BPRS. No significant changes were observed in the Activation subscale (Fig. 2; see Supplementary Figure, Supplemental Digital Content 5, which shows score changes in the BPRS subscales for each patient individually, http://links.lww.com/JCP/A348).

\section{Ayahuasca Effects on Blood Perfusion}

Ayahuasca administration was associated with significant $(P<0.01$, corrected $)$ activation of the left nucleus accumbens (peak intensity $=7.179$; voxels $=45 ; \mathrm{MNI}=-8,6,-10)$, right insula (peak intensity $=4.695$; voxels $=43 ; \mathrm{MNI}=32,24,-10$ ) and left subgenual area (peak intensity $=4.523$; voxels $=30$; $\mathrm{MNI}=-10,32,-8)($ Fig. 3).

\section{Tolerability}

Ayahuasca was well tolerated. Blood pressure and heart rate were nonsignificantly increased (data not shown). Vomiting, reported by $47 \%$ of the volunteers, was the only adverse effect recorded. Ayahuasca effects on thought content and sensory perception were considered mild and short-lived, and no dysphoric effects were reported. Volunteers were calm and relaxed during acute drug effects, and considered the ayahuasca session as a pleasant experience.

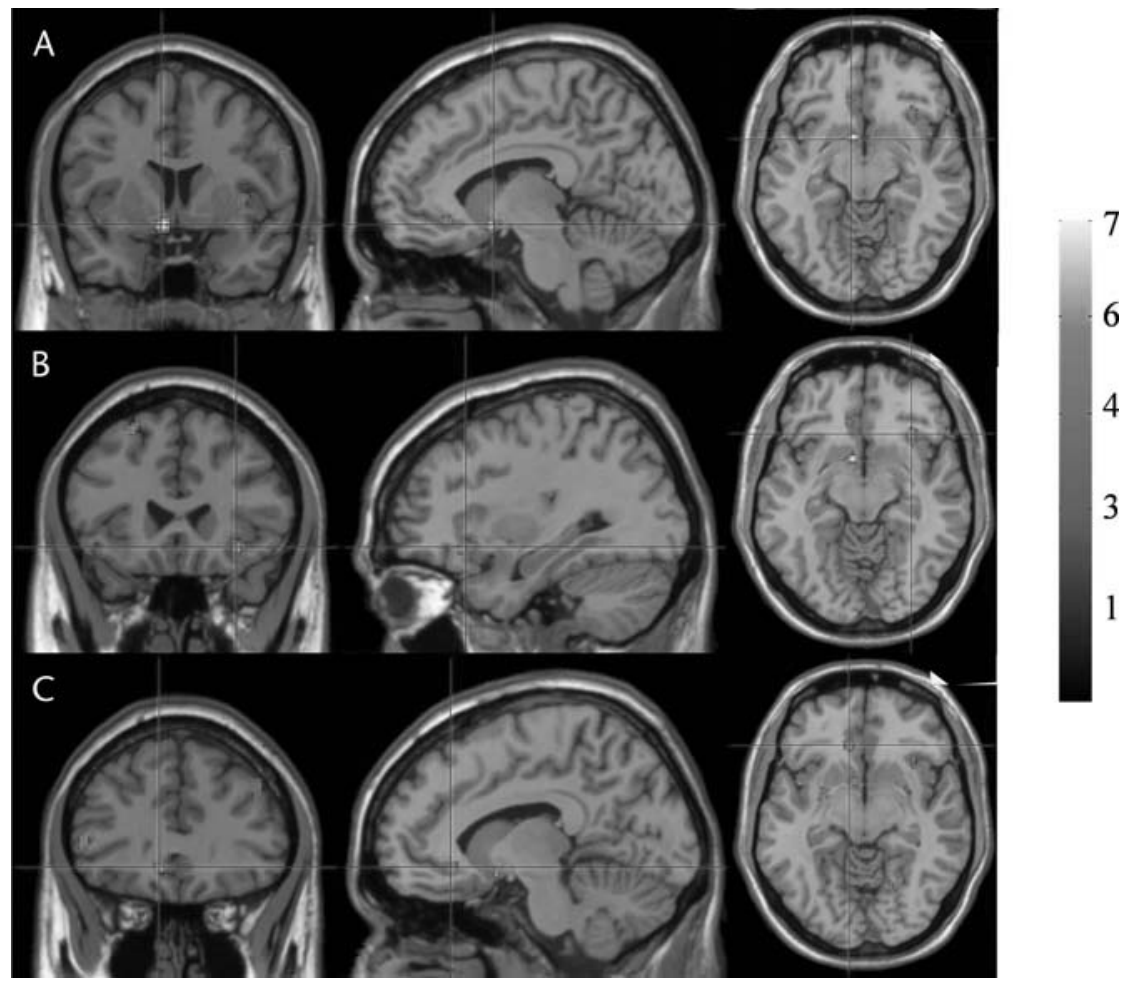

FIGURE 3. Statistical parametric maps of increases in regional cerebral blood flow in each of the 3 clusters showing suprathreshold voxels (exhibited in neurological convention). Each cluster is shown in the 3 orthogonal views (left, coronal; middle, sagittal; right, transverse) through the voxel with the maximum peak intensity value. MNI coordinates $(x, y$, and $z)$ and the number of voxels in each cluster are provided. $A$, Left nucleus accumbens $(-8,6-10$; voxels $=45$; peak intensity $=7.179)$. B, Right insula $(32,24,-10$; voxels $=43$, peak intensity $=4.695)$. $C$, Left subgenual cingulate cortex $(-10,32,-8$; voxels $=30$, peak intensity $=4.523)$. Results are shown at a $P$ value less than 0.01 , corrected 


\section{DISCUSSION}

Administration of ayahuasca was associated with rapid and sustained antidepressive effects. Results were similar across volunteers, regardless of the severity of the current depressive episode. Ayahuasca administration was also associated with increased blood perfusion in the nucleus accumbens, insula, and subgenual area, brain regions involved in the regulation of mood and emotional states.

Significant score decreases in HAM-D, MADRS, and Anxious-Depression BPRS subscale scores were observed during acute drug effects and from D1 to D21 and were related to depressed mood, sadness, anxiety, feelings of guilt, suicidal ideation, difficulties at work/activities, pessimistic thinking, and difficulty concentrating. Improvements in emotional withdrawal and blunted affect were (Thinking Disorder and Withdrawal-Retardation BPRS subscales) were also observed. Ayahuasca induced significant score increases in the CADSS scale at 40 to 80 minutes, suggesting increased psychoactivity in the same time point previously associated with peak ayahuasca psychoactive effects and DMT plasma levels. ${ }^{1-3}$

No significant changes in YMRS and Activation BPRS subscale scores were observed after ayahuasca intake, suggesting an absence of maniac-like effects. However, it is important to note that in our study a diagnosis of bipolar disorder and a previous history of mania or hypomania induced by antidepressant or substance use were considered exclusion criteria. Ayahuasca may be contraindicated for bipolar disorders patients, since a recent case report described a man with bipolar disorder that developed a manic episode after ayahuasca consumption. ${ }^{12}$

Increased blood perfusion in the subgenual area, nucleus accumbens, and insula were observed after ayahuasca intake. Hypoactivation of these brain regions is usually associated with depression, while increased activation is usually associated with antidepressive effects. ${ }^{13-17}$ Interestingly, ayahuasca increased blood perfusion in the anterior insula of healthy volunteers, but no significant changes were observed in the subgenual area or nucleus accumbens. ${ }^{3}$ These data suggest that our results may be specific to depressive patients.

The potential antidepressant effects of ayahuasca appear to be mediated by the agonist action of DMT on $5-\mathrm{HT}_{1 \mathrm{~A} / 2 \mathrm{~A} / 2 \mathrm{C}}$ receptors, since agonists of these receptors produce anxiolytic and antidepressive effects. ${ }^{18,19}$ Indeed, administration of DMT and other $5-\mathrm{HT}_{1 \mathrm{~A} / 2 \mathrm{~A} / 2 \mathrm{C}}$ receptor agonists such as psilocybin and LSD is associated with increases in positive mood in healthy volunteers $^{20-22}$ and in patients suffering life-threatening diseases. $^{23,24}$ Functional alterations in the default mode network $(\mathrm{DMN})$, a group of brain regions associated with self-perception, could also mediate the observed effects. Rumination, a core depressive symptom, is associated with increased DMN activity, and ayahuasca ${ }^{25}$ and psilocybin ${ }^{26}$ may reduce this activity. ${ }^{25}$ Theoretically, by changing one's self-perception, hallucinogens may reduce excessive attention to repetitive and pathological thoughts. ${ }^{1,25,26}$ Interestingly, decreases in brain blood flow observed in functional magnetic resonance imaging (fMRI) studies ${ }^{25,26}$ are inconsistent with our results and previous SPECT/positron emission tomography (PET) studies. ${ }^{1,3,27}$ These discrepancies may be related to different timescales involved in fMRI and SPECT/PET studies, which use radiotracers with long half-lives. Thus, phasic or short-term decreases in blood flow measured with fMRI may show some rebound effects and longer-term changes that are detected by SPECT/PET. ${ }^{26}$

Ayahuasca was well tolerated. No significant cardiovascular effects were observed after ayahuasca intake, and vomiting was the only adverse effect recorded. However, patients did not consider vomiting as causing severe discomfort. Moreover, ayahuasca psychoactive effects were considered mild and short-lived, and no dysphoric effects were reported. Indeed, volunteers were calm and relaxed under the effects of ayahuasca, considering it as a pleasant experience.

Although described results are promising, because treatment was not randomized or double-blind, and there was no placebo or other comparator group, we cannot conclude that the observed changes were in fact caused by ayahuasca. Moreover, it is important to note that the controlled clinical setting in which the experiments took place is different from the typical ritual context of ayahuasca consumption, which may impact the generalizability of our findings. ${ }^{6,28}$ Our results should be replicated in randomized, double-blind, placebo-controlled trials, and future research should investigate whether or how a ceremonial/ritual context may impact therapeutic outcomes.

\section{AUTHOR DISCLOSURE INFORMATION}

This research was conducted at the Department of Neurosciences and Behavior, Ribeirão Preto Medical School, University of São Paulo, Ribeirão Preto, Brazil. RFS is Fellow of the "Science without Borders" Program, Brazil (process no. 245447/2012-1). RGS is Fellow of the "National Post-Doctorate" Program, Brazil (PNPD/CAPES). For the remaining authors none were declared.

\section{REFERENCES}

1. McKenna D, Riba J. New World tryptamine hallucinogens and the neuroscience of ayahuasca. Curr Top Behav Neurosci. 2015; in press.

2. Riba J, Valle M, Urbano G, et al. Human pharmacology of ayahuasca: subjective and cardiovascular effects, monoamine metabolite excretion, and pharmacokinetics. J Pharmacol Exp Ther. 2003;306:73-83.

3. Riba J, Romero S, Grasa E, et al. Increased frontal and paralimbic activation following ayahuasca, the pan-Amazonian inebriant. Psychopharmacology (Berl). 2006;186:93-98.

4. dos Santos RG, Osório FL, Crippa JA, et al. Antidepressive and anxiolytic effects of ayahuasca: a systematic literature review of animal and human studies. Rev Bras Psiquiatr. 2015; in press.

5. Osório FL, Sanches RF, Macedo LRH, et al. Antidepressant effects of a single dose of ayahuasca in patients with recurrent depression: a preliminary report. Rev Bras Psiquiatr. 2015;37:13-20.

6. Labate BC, Cavnar C (Eds). The therapeutic use of ayahuasca. Berlin: Springer; 2014

7. Del-Ben CM, Vilela JAA, Crippa JAS, et al. Reliability of the structured clinical interview for DSM-IV - clinical version translated into Portuguese. Rev Bras Psiquiatr. 2001;23:156-159.

8. Moreno RA, Moreno DH. Hamilton (HAM-D) \& Montgomery \& Asberg (MADRS) depression rating scales. Rev Psiquiatr Clín. 1998;25:262-272.

9. Vilela JA, Crippa JA, Del-Ben CM, et al. Reliability and validity of a Portuguese version of the Young Mania Rating Scale. Braz J Med Biol Res. 2005;38:1429-1439.

10. Crippa JA, Sanches RF, Hallak JE, et al. Factor structure of Bech's version of the Brief Psychiatric Rating Scale in Brazilian patients. Braz J Med Biol Res. 2002;35:1209-1213.

11. Hallak JE, Dursun SM, Bosi DC, et al. The interplay of cannabinoid and NMDA glutamate receptor systems in humans: preliminary evidence of interactive effects of cannabidiol and ketamine in healthy human subjects. Prog Neuropsychopharmacol Biol Psychiatry. 2011;35:198-202.

12. Szmulewicz AG, Valerio MP, Smith JM. Switch to mania after ayahuasca consumption in a man with bipolar disorder: a case report. Int J Bipolar Disord. 2015;3:4

13. Drevets WC, Savitz J, Trimble M. The subgenual anterior cingulate cortex in mood disorders. CNS Spectr. 2008;13:663-681. 
14. Pizzagalli DA, Holmes AJ, Dillon DG, et al. Reduced caudate and nucleus accumbens response to rewards in unmedicated individuals with major depressive disorder. Am J Psychiatry. 2009;166:702-710.

15. Fitzgerald PB, Laird AR, Maller J, et al. A meta-analytic study of changes in brain activation in depression. Hum Brain Mapp. 2008;29:683-695.

16. Bewernick BH, Hurlemann R, Matusch A, et al. Nucleus accumbens deep brain stimulation decreases ratings of depression and anxiety in treatment-resistant depression. Biol Psychiatry. 2010;67:110-116.

17. Merkl A, Schneider GH, Schönecker T, et al. Antidepressant effects after short-term and chronic stimulation of the subgenual cingulate gyrus in treatment-resistant depression. Exp Neurol. 2013;249:160-168.

18. Masuda Y, Sugiyama T. The effect of globopentaosylceramide on a depression model, mouse forced swimming. Tohoku J Exp Med. 2000;191:47-54

19. Nic Dhonnchadha BA, Hascoët M, Jolliet P, et al. Evidence for a 5-HT $2 \mathrm{~A}$ receptor mode of action in the anxiolytic-like properties of DOI in mice. Behav Brain Res. 2003;147:175-184.

20. Riba J, McIlhenny EH, Bouso JC, et al. Metabolism and urinary disposition of $N, N$-dimethyltryptamine after oral and smoked administration: a comparative study. Drug Test Anal. 2015;7:401-406

21. Kraehenmann R, Preller KH, Scheidegger M, et al. Psilocybin-induced decrease in amygdala reactivity correlates with enhanced positive mood in healthy volunteers. Biol Psychiatry. 2015;78:572-581; in press.
22. Schmid Y, Enzler F, Gasser P, et al. Acute effects of lysergic acid diethylamide in healthy subjects. Biol Psychiatry. 2015;78:544-553; in press.

23. Grob CS, Danforth AL, Chopra GS, et al. Pilot study of psilocybin treatment for anxiety in patients with advanced-stage cancer. Arch Gen Psychiatry. 2011;68:71-78.

24. Gasser P, Holstein D, Michel Y, et al. Safety and efficacy of lysergic acid diethylamide-assisted psychotherapy for anxiety associated with life-threatening diseases. J Nerv Ment Dis. 2014;202:513-520.

25. Palhano-Fontes F, Andrade KC, Tofoli LF, et al. The psychedelic state induced by ayahuasca modulates the activity and connectivity of the default mode network. PLoS One. 2015;10:e0118143.

26. Carhart-Harris R, Erritzoe D, Williams T, et al. Neural correlates of the psychedelic state as determined by fMRI studies with psilocybin. Proc Natl Acad Sci U S A. 2012;109:2138-2143

27. Vollenweider FX, Leenders KL, Scharfetter C, et al. Positron emission tomography and fluorodeoxyglucose studies of metabolic hyperfrontality and psychopathology in the psilocybin model of psychosis. Neuropsychopharmacology. 1997;16:357-372.

28. Tupper KW, Labate BC. Ayahuasca, psychedelic studies and health sciences: the politics of knowledge and inquiry into an Amazonian plant brew. Curr Drug Abuse Rev. 2014;7:71-80. 